\title{
Measurement of Backscattered GPS Signals
}

\author{
Tore Lindgren*, Eric Vinande ${ }^{\dagger}$, Dennis Akos ${ }^{\dagger}$, Dallas Masters ${ }^{\dagger}$, and Penina Axelrad ${ }^{\dagger}$ \\ *Luleå University of Technology, Luleå, Sweden \\ †University of Colorado, Boulder, Colorado, USA
}

\begin{abstract}
This paper describes a technique utilizing GPS ground reflections (GPS bistatic radar) to detect objects with a significant radar cross section located on the surface of the earth. GPS bistatic radar has been shown to be effective as a radar altimeter and for characterization of the reflection surface but has thus far not been shown to be effective for object detection. The technique uses ground reflections with longer path delay than the shortest path specular reflection. Data was collected using a COTS software receiver and post-processed using an in-house tool. Regions with ground reflections were overlayed on aerial imagery to identify possible sources.
\end{abstract}

\section{INTRODUCTION}

The use of GPS signals as a passive radar system is becoming increasingly popular as an alternative to radar altimetry. Previously, GPS bistatic radar has been used for aircraft altimetry [1]-[3], remote sensing of ocean parameters [4], measurement of soil moisture content [3], and object detection [2]. Thus far, the focus has been on using the shortest path specular reflection.

This paper presents results from an airborne GPS bistatic radar experiment. The focus is on how this method can be used as a means of detecting objects on the ground. The technique described make use of further delayed reflections to detect objects. In this section a summary of the data collection campaign and an introduction to the GPS bistatic radar concept is given.

\section{A. Bistatic GNSS Radar Concept}

Unlike monostatic radar where a single system transmits and receives reflected radio frequency $(\mathrm{RF})$ energy, bistatic radar uses geographically separated radar transmitters and receivers. In GPS bistatic radar the transmitter is the GPS satellite and the receiver is located on another satellite or airborne platform. This technique compares the direct and ground-reflected signals received from the GPS satellite for remote sensing purposes. This is shown in Figure 1 where a reflecting object is also present. The correlation delay between the two paths when combined with the GPS satellite elevation angle gives a measure of the aircraft's height above ground level (AGL). The strength and shape of the reflected signal's correlation waveform provides insight into the ground surface's reflection properties.

\section{B. Data Collection Summary}

During data collection campaigns over the summers of 2004 and 2005, researchers at the University of Colorado (CU), Boulder, digitally sampled the direct and reflected GPS

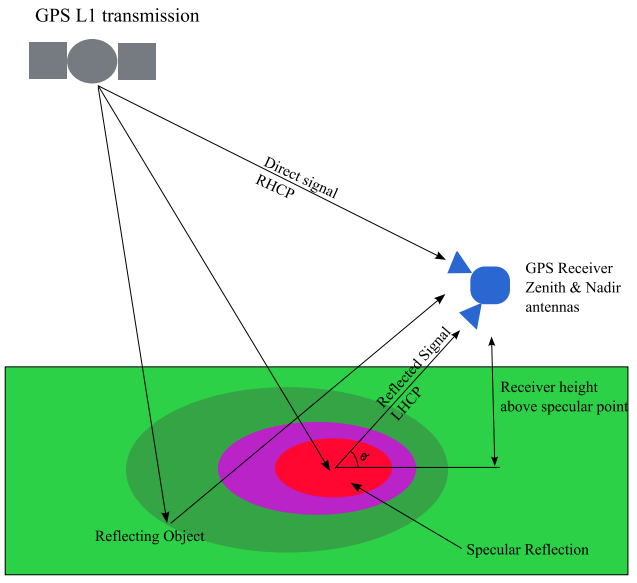

Fig. 1. Bistatic GPS radar concept with a GPS satellite, specular area, reflecting object, and a GPS receiver.

spectrum from an airborne platform using zenith- and nadirpointing antennas. These campaigns were conducted in support of ongoing bistatic radar research at $\mathrm{CU}$. The focus of this paper is data collected on July 1, 2005, over Iowa. Figure 2 shows the ground track of this test flight. The total length of this measurement is 102 minutes. A skyplot with the satellites analyzed is shown in Figure 3.

Raw RF samples were collected using a dual input NordNav software receiver from both the direct- and ground-reflected signals with zenith- and nadir-pointing antennas. This data collection campaign is described in detail in [5]. The data from the direct channel provided information about the aircraft's position and velocity. The ground reflections were tracked by slaving the reflected channels's code and carrier tracking loop parameters to the direct channel.

During post-processing, additional correlators were added with 0.1 chip spacing from -1.1 to 16.1 chips delay on the reflected channel to cover the delay for which the reflections were expected to produce a correlation waveform. This corresponds to a ground distance of up to approximately $9 \mathrm{~km}$ for an aircraft at an altitude of $582 \mathrm{~m}$ and a satellite at $58^{\circ}$ elevation, but can change significantly for different elevation angles, see Section II-B for a more detailed analysis. Along with the expected shortest path specular point reflections, instances of further delayed reflections, or secondary reflections, were discovered. These backscattered reflections were transient, thus implicating them as reflections off structures, vehicles, etc. only when the geometry was correct between the GPS satellite, aircraft, and reflecting body. Figure 4 shows the 


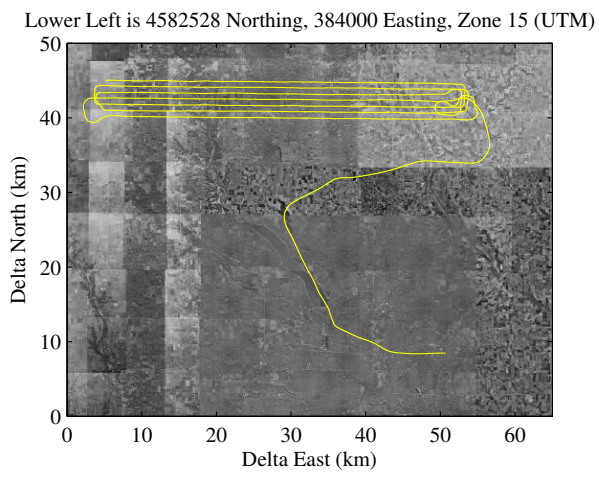

Fig. 2. Flight test ground track. Image courtesy of the USGS [6].

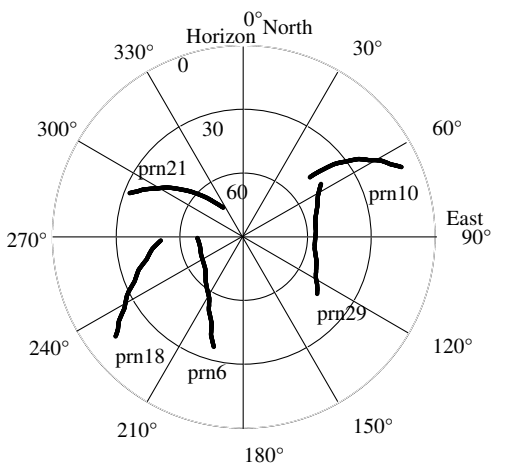

Fig. 3. Sky plot of selected satellites.

correlation waveform for the direct and reflected channels normalized to the maximum value of the direct channel. The direct signal (left) is phase locked and since no reflected signals are expected on this channel the correlation waveform is only shown between -1 and 1 chip. A clearly visible secondary peak with a delay of about 5 chips can be seen in addition to the specular reflection (with a delay of about 2 chips). The chipping rate is $1.023 \mathrm{MHz}$ which gives a wavelength (chip width) of $293.05 \mathrm{~m}$. The small peak at 0 chip delay is the direct signal leaking in to the reflected channel. This could be either signal propagating around the aircraft body or cross talk in the front end. This does not have a major contribution to the results due to the delay between the direct and reflected signals. By studying the strength and duration of the secondary reflection it is possible to get insight into the reflecting object's characteristics and size.

\section{DATA ANALYSIS}

\section{A. Peak Detection and Threshold}

The object of this study is to examine the possibility of using secondary reflections to detect object on the ground. Since it is an initial study, only the strongest and most obvious secondary reflections have been considered. The conditions used are therefore strict and selected in order to eliminate the risk of mistaking noise for actual secondary reflections. Thus,
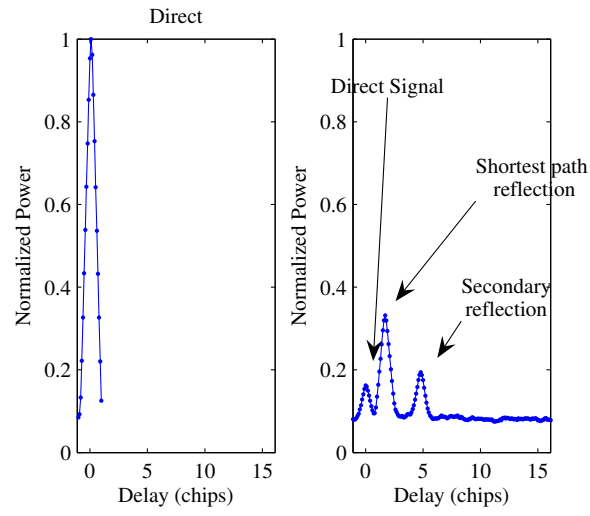

Fig. 4. Correlation waveforms for direct (left) and reflected (right) channels.

there is a significant potential for refinement of these. The conditions used can be summarized as:

1) Strength of Secondary Reflection: The first and most obvious criteria is the strength of the secondary reflection compared to the average noise. By manual inspection of the data it was found that a strength of $5 \sigma$ (5 times the standard deviation of the noise) above the noise floor is suitable (see Figure $5 \mathrm{~B}$ ). As noise floor an average of the correlation waveform between 5.8 and 16.1 chips was used. This was chosen to include some reflected power but no significant secondary reflections.

2) Secondary Peak Clearly Distinguishable from Shortest Path Reflection: To simplify the detection of secondary reflections and the subsequent analysis only those peaks clearly distinguishable from the shortest path reflection were considered. To achieve this, the minimum value between the two peaks must be at least $5 \sigma$ below the secondary reflection (see Figure $5 \mathrm{~A}$ ), $\sigma$ was defined in the same way as previously.

3) Strong Specular Reflection: A strong shortest path reflection was also required. An arbitrary threshold of $15 \sigma$ above the noise floor was set for this reflection, $\sigma$ and the noise floor was defined in the same way as previously. This reflection also had to be stronger than the direct signal leakage on the reflected channel.

4) Continuity: The same reflection had to be seen in at least two consecutive epochs. This reduced the risk of having reflections of vehicles and small objects.

\section{B. Position of Reflecting Object}

By looking at a secondary reflection from one satellite it is possible to determine the coordinates on the ground where this could have come from. The information needed to do this is the direction vector of the satellite (azimuth and elevation) and the height above ground level (AGL) of the measurement platform, in this case the aircraft. A good approximation of the AGL is given in [2],

$$
h \approx \frac{\delta_{1}}{2 \sin \theta_{e l}},
$$

where $h$ is the AGL, $\delta_{1}$ is the path delay of the shortest path reflection (see Figure 6) and $\theta_{e l}$ is the elevation angle 


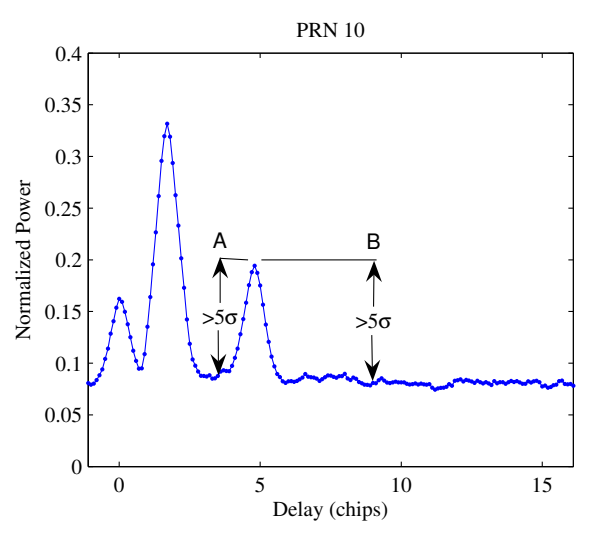

Fig. 5. Threshold used when detecting secondary reflections.

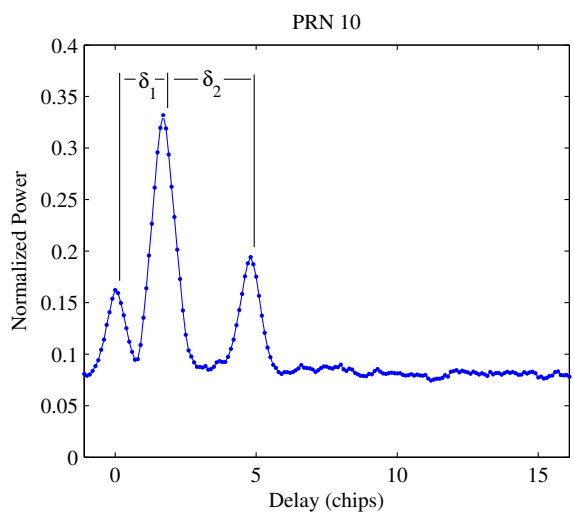

Fig. 6. Path delay of shortest path reflection, $\delta_{1}$, and path delay between shortest path reflection and secondary reflection, $\delta_{2}$.

of the satellite. The specular point can also be calculated using a digital elevation map [3]. This gives a more accurate estimation of AGL than the one given in (1). It was, however, only done for one satellite (PRN 29). By using the path delay $\delta_{2}$ between the secondary and specular reflection (see Figure 6) it is possible to calculate possible coordinates of these reflections. It turns out that this is an ellipse given by

$$
\frac{x^{2}}{a^{2} \sin ^{2} \theta_{e l}}+\frac{\left(y-y_{c}\right)^{2}}{a^{2}}=1,
$$

where

$$
\begin{gathered}
y_{c}=\frac{\delta_{2}+h \sin \theta_{e l}}{\sin \theta_{e l} \tan \theta_{e l}} \\
a=\frac{\sqrt{\delta_{2}^{2}+2 \delta_{2} h \sin \theta_{e l}}}{\sin ^{2} \theta_{e l}},
\end{gathered}
$$

$z$ is calculated from (1). In (2) a flat surface has been assumed and the elevation and azimuth of the satellite has been assumed to be the same at both the aircraft and the reflecting object (see Figure 7). Also, the azimuth is in the direction of the positive $y$-axis.

Figure 8 shows the path delay for reflections of different positions on the ground. The aircraft is at $(x, y)=(0,0)$ and the AGL is $232 \mathrm{~m}$, which is the average AGL of the aircraft

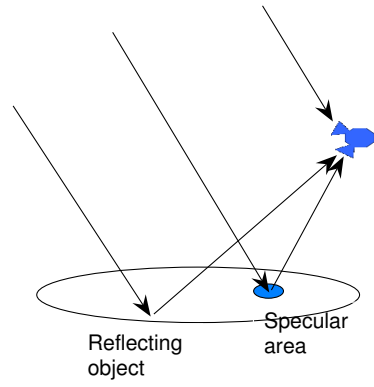

Fig. 7. Geometry between direct signal, specular reflections, and secondary reflection of an object.

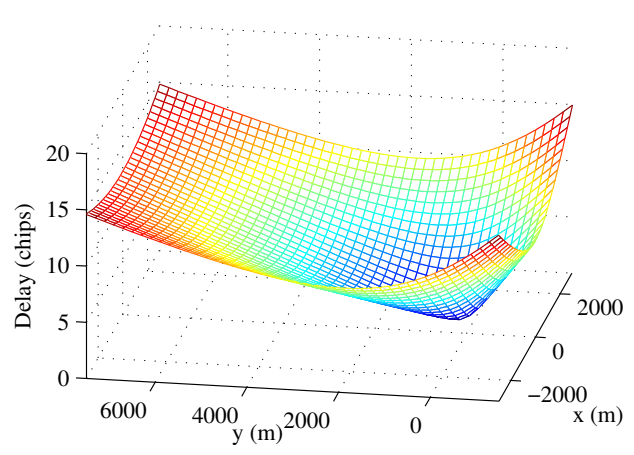

Fig. 8. Path delay vs. position on ground. The elevation of the satellite is $58^{\circ}$, pointing in the direction of the positive $y$-axis

used for the data collection. The elevation of the satellite was set arbitrarily to $58^{\circ}$, pointing in the direction of the positive y-axis (see Figure 8). It can be seen that the path delay increases more rapidly for signals reflected from behind the aircraft (as seen from the satellite) than for signals reflected off objects in front of the aircraft.

\section{RESULTS}

The analysis described in the previous section was done for five satellites with relatively high elevation angles, PRN 6, 10, 18,21 , and 29. How the results depend on the averaging time used and some examples of detected objects are presented in the following two subsections. The last subsection presents some interesting results not analyzed in detail here but that are important and should be included in the future.

\section{A. Averaging Time}

Since the measurement platform is dynamic the characteristics of the secondary reflections found will depend heavily on the averaging time. The average speed of the aircraft was $75 \mathrm{~m} / \mathrm{s}$ and the average altitude was $582 \mathrm{~m}$. For large averaging times the aircraft has time to move a considerable distance during the averaging and thus, only large objects should be detected and many of the smaller objects seen using shorter averaging times will not be seen. The noise seen in the 
TABLE I

GROUND COVERED BY AN AIRBORNE MEASUREMENT PLATFORM

\begin{tabular}{|c|c|c|c|}
\hline Averaging time & $10 \mathrm{~m} / \mathrm{s}$ & $75 \mathrm{~m} / \mathrm{s}$ & $150 \mathrm{~m} / \mathrm{s}$ \\
\hline $20 \mathrm{~ms}$ & $0.2 \mathrm{~m}$ & $1.5 \mathrm{~m}$ & $3 \mathrm{~m}$ \\
\hline $300 \mathrm{~ms}$ & $3 \mathrm{~m}$ & $22.5 \mathrm{~m}$ & $45 \mathrm{~m}$ \\
\hline $800 \mathrm{~ms}$ & $8 \mathrm{~m}$ & $60 \mathrm{~m}$ & $120 \mathrm{~m}$ \\
\hline
\end{tabular}

correlation waveforms should be considerably lower than for shorter averaging times which makes it easier to detect objects. Figure 9 shows the total number of secondary reflections found in the data set (for the satellites analyzed) as a function of averaging time. It can be seen that the number of secondary reflections decreases rapidly for high averaging times (a). A tendency to decreasing number of secondary reflections can also be seen in (b) for low averaging times. A maximum occurs for averaging times between 20 and $30 \mathrm{~ms}$, which corresponds to a movement of the aircraft of 1.5 to $2 \mathrm{~m}$. Table I shows the distance traveled for some different aircraft speeds and averaging times. The speeds were chosen to be representative for some different aircrafts, $75 \mathrm{~m} / \mathrm{s}$ was the average speed of the aircraft used in this data collection and $10 \mathrm{~m} / \mathrm{s}$ is a typical speed for a high-altitude balloon. The number of objects detected is expected to increase significantly if the averaging time could be increased with respect to covered ground. A slow moving or static platform would therefore be a more efficient solution.

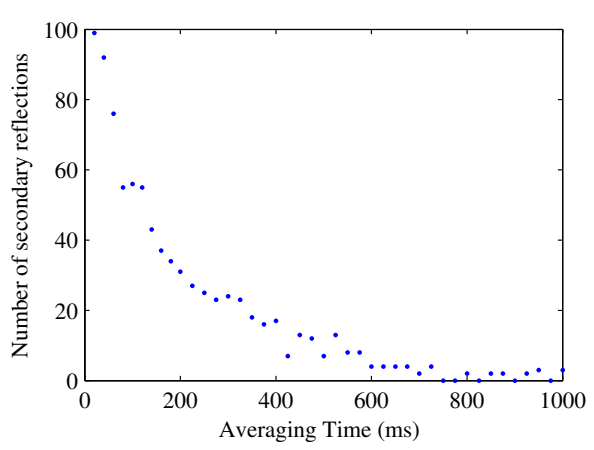

(a)

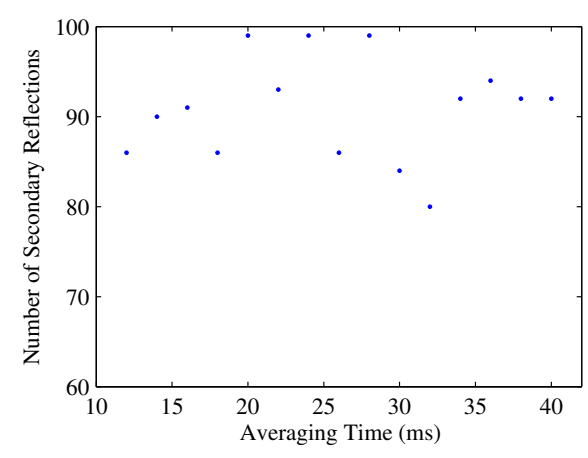

(b)

Fig. 9. Number of secondary reflection found versus averaging time.
Of interest is also to study how these secondary reflections are distributed for different averaging times. This is done in Figure 10 for $20 \mathrm{~ms}$ (a), $300 \mathrm{~ms}$ (b), and $800 \mathrm{~ms}$ (c) averaging times. The colored ellipses correspond to the area where the reflecting object can be found for a given PRN (see Section IIB). In (a) the secondary reflections are fairly well distributed while they are concentrated to the lower part of the flight in (b) and (c). This is due to a larger number of large structures in this part.

\section{B. Detection of Objects}

1) Urban Reflections: In Figure 11 the possible sources of secondary reflections are shown for an urban area, in this case Des Moines, Iowa. The averaging time is $20 \mathrm{~ms}$, the color of the ellipse represents the same satellites as in Figure 10. As expected, the ellipse representing the possible sources of secondary reflections intersects with many structures. It is therefore not realistic to determine which building is the reflector.

2) Rural Reflections: Of greater interest is to study the secondary reflections in a rural area. This is shown in Figure 12 (for PRN 21). The averaging time is $20 \mathrm{~ms}$. The specular point is shown as a colored ' + ' and the position of the aircraft as a yellow ' $x$ '. It can be seen that the ellipse representing a possible secondary reflection intersects with a farm in this figure. There are more than one possible source in this case and from the imagery available it is not possible to determine which is the actual source of the reflection. To identify a unique object on the ground this would have to be seen in several different satellites. The ellipses representing the area where the reflection could come from would then intersect over the object, reflections from three satellites would give a unique position of the object. No instances of this was found, probably due to the fact that only the strongest reflections have been analyzed. The geometry between the satellite, reflecting object, and aircraft is not likely optimal for different satellites.

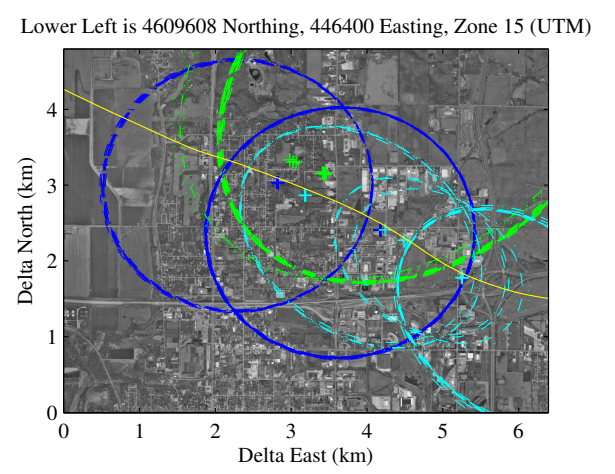

Fig. 11. Possible reflecting objects in an urban area (Des Moines, Iowa). The color of the ellipse represents the same satellites as in Figure 10. Image courtesy of the USGS [6]. 


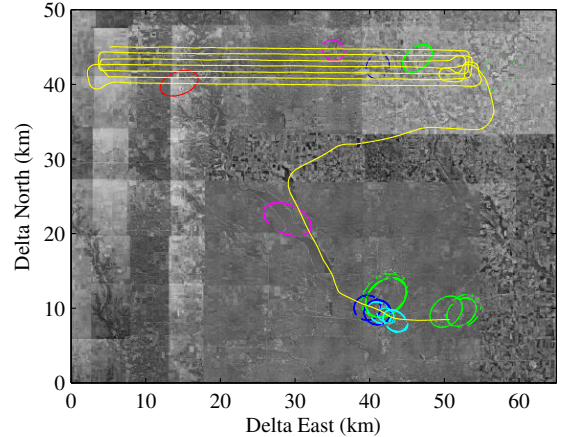

(a) $20 \mathrm{~ms}$ averaging

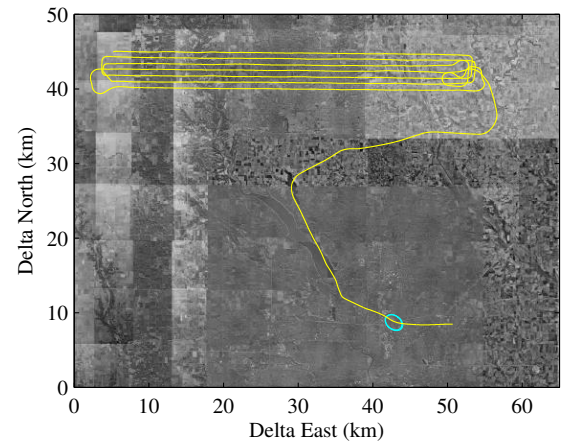

(c) $800 \mathrm{~ms}$ averaging

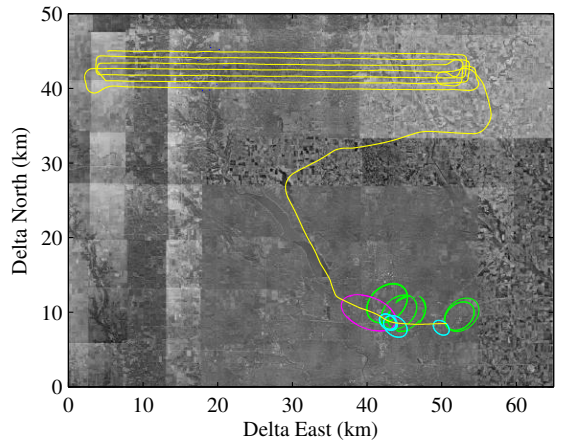

(b) $300 \mathrm{~ms}$ averaging

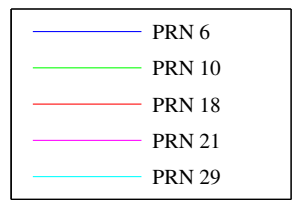

Fig. 10. Secondary reflections overlayed on aerial imagery for different averaging times. Image courtesy of the USGS [6].

\section{Multiple Reflections and Short Path Delay}

Instances of multiple secondary reflections was also seen, which is shown in Figure 13. This was only seen for one satellite (PRN 10) and only for averaging times lower than $80 \mathrm{~ms}$. Also, it was only seen during a short period in the beginning of the data set, over the urban area. In this analysis, only one peak was used. These multiple reflections would, however, be more frequent if the threshold were set lower.

If the secondary reflection has a short path delay relative the specular reflection the two peaks can be difficult to distinguish, and the path delay is also harder to estimate. This was found in several cases, one example (PRN 6) is shown in Figure 14. The averaging time is here $20 \mathrm{~ms}$. Since these reflections have a shorter path delay they can be expected to be significantly stronger than reflections occuring farther away. These have not been analyzed here but should be included in future analyzes.

\section{CONCLUSION}

A method of detecting objects on the ground using secondary reflections with GPS bistatic radar have been presented. The focus has been on finding a possible source of the reflection given a path delay of the signal. The possible sources of a secondary reflection are represented by an ellipse. By using reflections from multiple satellites it should be possible to find a more accurate position estimate of the source. Since only the most obvious secondary reflections has been analyzed effort should be put into finding weaker reflections. This can be done by using a high-gain antenna and by having a slower moving or static measurement platform, enabling longer averaging times. By taking a static measurement (e.g. from a tall tower instead of an aircraft) both the geometry and the possibility of finding weaker signals could be studied under controlled circumstances.

\section{ACKNOWLEDGMENT}

This work was funded in part under Cooperative Agreement NNL04AA59G through the NASA Langley Research Center. Any opinions, findings, and conclusions or recommendations expressed in this material are those of the authors and do not necessarily reflect the views of the National Aeronautics and Space Administration.

The authors would also like to thank the researchers at University of Iowa who helped during the data collection campaign. 


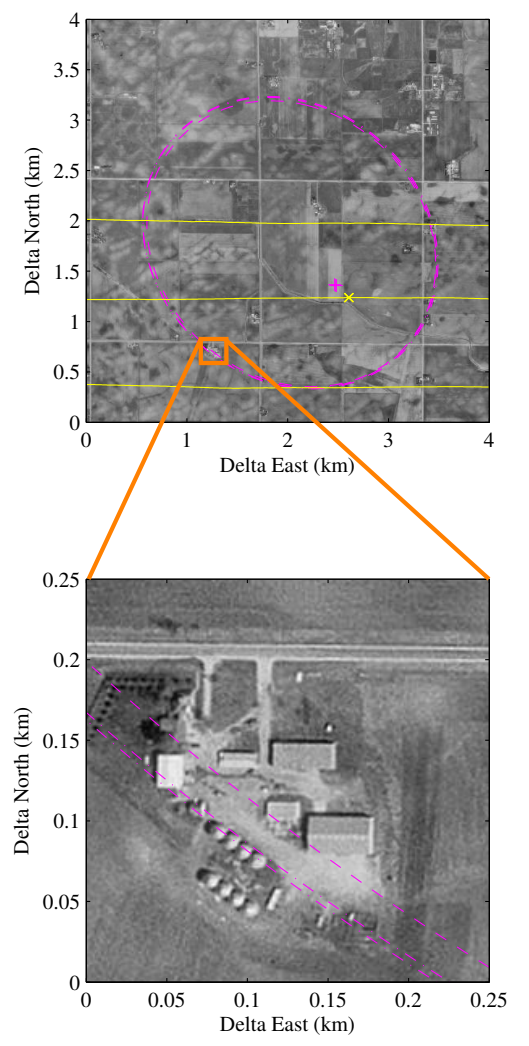

Fig. 12. Possible source of secondary reflection (PRN 21). The specular point is shown as a colored ' + ' and the position of the aircraft as a yellow ' $x$ '. Image courtesy of the USGS [6].

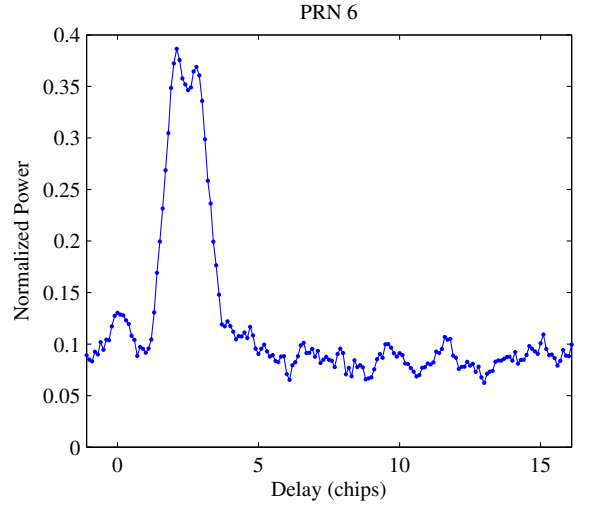

Fig. 14. Closely spaced peaks.

\section{REFERENCES}

[1] D. Masters, P. Axelrad, V. Zavorotny, S. J. Katzberg, and F. Lalezari, A passive GPS bistatic radar altimeter for aircraft navigation, Proceedings of the Institute of Navigations ION GPS-2001, Salt Lake City, Utah, September, 2001.

[2] E. Vinande, D. M. Akos, and D. Masters, GPS bistatic radar measurements of aircraft altitude and ground objects with a software receiver, Proceedings of the Institute of Navigations 61st Annual Meeting, Cambridge, Massachusetts, June, 2005.

[3] D. Masters, Surface Remote Sensing Applications of GNSS Bistatic Radar: Soil Moisture and Aircraft Altimetry, Ph.D. dissertation, Aerospace Engineering Sciences, Boulder, Colorado, December, 2004.

[4] M. Armatys, D. Masters, A. Komjathy, P. Axelrad, and J. L. Garrison, Exploiting GPS as a new oceanographic remote sensing tool, 3rd ed. Proceedings of the Institute of Navigations National Technical Meeting 2001, Long Beach, California, 2001.

[5] S. Eterhuizen, D. Masters, D. M. Akos, and E. Vinande, Experimental characterization of land-reflected GPS signals, Proceedings of the Institute of Navigations ION GNSS-2005, Long Beach, California, September, 2005.

[6] TerraServer-USA (2006, April 18). [Online]. Available: http://www.terraserverusa.com.

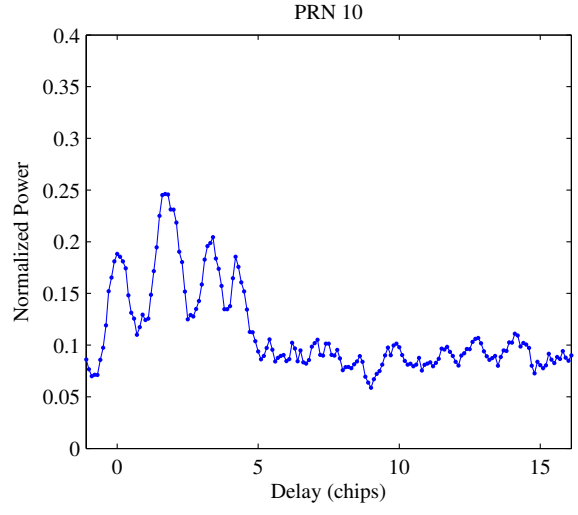

Fig. 13. Multiple secondary reflections. 